\title{
Correction: Excessive Smartphone Use and Self-Esteem Among Adults With Internet Gaming Disorder: Quantitative Survey Study
}

\author{
Hyunmin $\mathrm{Kim}^{1,2}, \mathrm{PhD}$; In Young $\mathrm{Choi}^{1}, \mathrm{PhD}$; Dai-Jin $\mathrm{Kim}^{3}, \mathrm{MD}, \mathrm{PhD}$
}

${ }^{1}$ Department of Medical Informatics, College of Medicine, The Catholic University of Korea, Seoul St. Mary's Hospital, Seoul, Republic of Korea

${ }^{2}$ Division of Health Systems Management and Policy, School of Public Health, The University of Memphis, Memphis, TN, United States

${ }^{3}$ Department of Psychiatry, College of Medicine, The Catholic University of Korea, Seoul St. Mary's Hospital, Seoul, Republic of Korea

\section{Corresponding Author:}

In Young Choi, $\mathrm{PhD}$

Department of Medical Informatics

College of Medicine

The Catholic University of Korea, Seoul St. Mary's Hospital

222 Banpo-daero, Seocho-gu

Seoul

Republic of Korea

Phone: 8222587870

Email: iychoi@catholic.ac.kr

\section{Related Article:}

Correction of: https://mhealth.jmir.org/2020/9/e18505/

(JMIR Mhealth Uhealth 2020;8(11):e24869) doi: 10.2196/24869

In "Excessive Smartphone Use and Self-Esteem Among Adults With Internet Gaming Disorder: Quantitative Survey Study" (JMIR Mhealth Uhealth 2020;8(9):e18505), the authors noted one error in the Acknowledgments section.

In the originally published manuscript, the final sentence of the Acknowledgments section read "This work was supported by the National Research Foundation of Korea (NRF) (grant no. 2014M3C7A1062893)." The has been changed to: "This work was supported by the National Research Foundation of Korea

$\begin{array}{lccc}\text { (NRF) (grant } & \text { nos. } & \text { 2014M3C7A1062893 and } \\ \text { NRF-2019R1A5A2027588)." } & & \end{array}$

The correction will appear in the online version of the paper on the JMIR Publications website on November 3, 2020, together with the publication of this correction notice. Because this was made after submission to PubMed, PubMed Central, and other full-text repositories, the corrected article has also been resubmitted to those repositories.

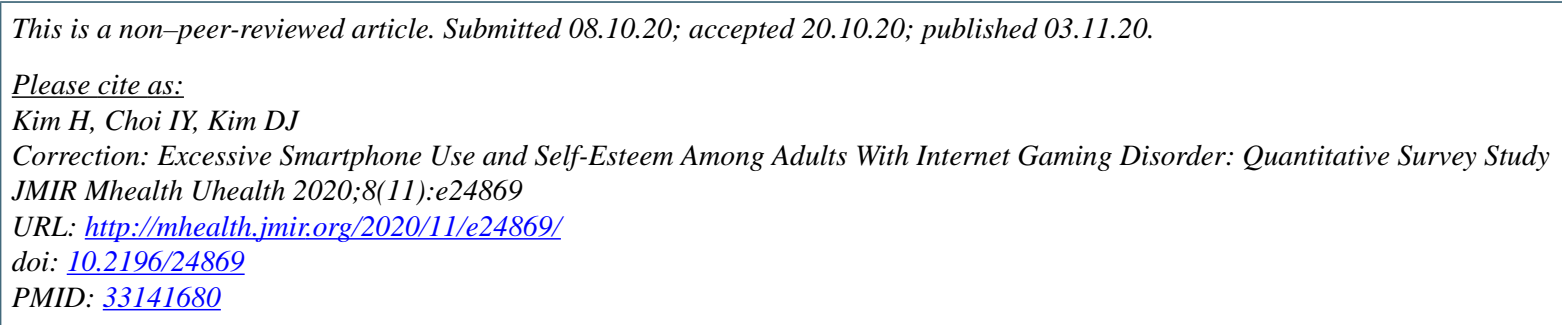

CHyunmin Kim, In Young Choi, Dai-Jin Kim. Originally published in JMIR mHealth and uHealth (http://mhealth.jmir.org), 03.11.2020. This is an open-access article distributed under the terms of the Creative Commons Attribution License (https://creativecommons.org/licenses/by/4.0/), which permits unrestricted use, distribution, and reproduction in any medium, provided the original work, first published in JMIR mHealth and uHealth, is properly cited. The complete bibliographic information, a link to the original publication on http://mhealth.jmir.org/, as well as this copyright and license information must be included. 